\title{
ON THE UNIFORMIZATION OF AN ALGEBRAIC FUNCTION OF GENUS $p \geqq 2$
}

\author{
Masatsugu Tsuji
}

(Received July 2, 1951)

1.

Picard ${ }^{1)}$ proved the following theorem.

TheOREM 1. Let $F$ be a closed Riemann surface of genus $p \geqq 2$ spread over the $x$-plane Then we can not uniformize $F$ by $x=x(t)$, which is onevalued and meromorphic in $0<|t| \leqq R$ and has an essential singularity at $t=0$.

Proof. Suppose that there exists a function $x=x(t)$, which satisfies the condition of the theorem. Since $F$ is of hyperbolic type, we can map the universal covering surface $F^{(\infty)}$ of $F$ on $|z|<1$ by $x=\varphi(z)$ and put $z=z(t)$. Then $z(t)$ is not one-valued in $0<|t| \leqq R$. For, if it is one-valued, then, since $|z(t)|<1$ in $0<|t|<R, z(t)$ is regular at $t=0$, so that $x=x(t)$ is meromorphic at $t=0$, which contradicts the hypothesis. Hence $z(t)$ is many valued, so that to a circle $C:|t|=\rho(<R)$, there corresponds a curve $L$ on $F$, which is not homotop null, hence the image of $C$ in $|z|<1$ is either (i) a Jordan curve, which has a common point $\gamma$ with $|z|=1$ or (ii) a Jordan arc, whose end points $\alpha, \beta(\alpha \neq \beta)$ lie on $|z|=1$. The case (i) does not occur. For, if it does occur, then $\lim _{t \rightarrow 0} z(t)=\gamma$, so that $\lim _{t \rightarrow 0} x(t)=x_{0}$ $=\varphi(\gamma)$, which contradicts the hypothesis, that $t=0$ is an essential singularity of $x(t)$. Hence the case (ii) occurs. Let $\alpha, \beta$ be so chosen that

$$
\lim _{\theta \rightarrow+\infty} z\left(\rho e^{i \theta}\right)=\alpha, \lim _{\theta \rightarrow-\infty} z\left(\rho e^{i \theta}\right)=\beta .
$$

By $u=\log t$, we map $0<|t| \leqq \rho$ on the half-plane $\Re u \leqq \log \rho$ and then by a linear transformation, we map this half-plane on $|\tau| \leqq 1$, such that $\tau=-1$ corresponds to $u=-\infty$ and put $z(t)=z(\tau)$, then $z(\tau)$ is regular and $|z(\tau)|<1$ in $|\tau|<1$. By (1)

$$
\lim _{\varphi \rightarrow \pi-0} z\left(e^{i \varphi}\right)=\alpha, \lim _{\varphi \rightarrow-a+0} z\left(e^{i \varphi}\right)=\beta \quad(\alpha \neq \beta) .
$$

which contradicts Lindelöf's theorem. Hence our theorem is proved.

2 .

Let $F$ be a Riemann surface and $F^{*}$ be its covering surface. If $F^{*}$ has no branch points relatively to $F$, then we call $F^{*}$ a non-ramified (unver$z$ weigt) covering surface of $F$.

TheOREM 2. Let $F$ be a closed Riemann surface of genus $p \geqq 2$ spread

1) E. PICARD : Démonstration d'un théorème géneral des fonctions uniformes liées par une relation algébrique. Acta. Math. 11(1887). 
over the $x$-sphere. Then there exists no function $x=x(t)$, which is one-valued and meromorphic in a neighbourhood $U$ of a closed set $E$ of logarithmic catacity zero, every point of which is an essential singularity of $x(t)$, such that the Riemdnn surface $F^{*}$ generated by $x=x(t)$ is a non-ramified covering surface of $F$.

We remark that in Theorem 1 , the Riemann surface $F^{*}$ generated by $x=x(t)$ is not supposed to be non-ramified relatively to $F$.

Proof. Suppose that there exists a function $x=x(t)$, which satisfieds the condition of the theorem, such that the Riemann surface $F^{*}$ generated by $x=x(t)$ is a non-ramified covering surface of $F$.

Since $E$ is a closed set of logarithmic capacity zero, by Evans' theorem ${ }^{2)}$, we can distribute a positive mass $d \mu(a)$ on $E$ of total mass 1 , such that

$$
u(t)=\int_{E} \log \frac{1}{|t-a|} d \mu(a), \quad\left(\int_{E^{\prime}} d \mu(a)=1\right)
$$

tends to $+\infty$, if $t$ tends to any point of $E$. Let $C_{r}$ be the niveau curve: $u(t)=r$, then $C_{r}$ consists of a finte number of Jordan curves, which cluster to $E$ as $r \rightarrow \infty$.

Let $\theta(t)$ be conjugate harmonic function of $u(t)$, then since the total mass is 1 ,

$$
\int_{C_{r}} d \theta(t)=2 \pi
$$

We put

$$
\tau=e^{u+i \theta}=r(t) e^{i \theta(t)}, \quad x=x(t)=x(\tau) .
$$

Let $A(r)$ be the area on the $x$-sphere of the image of the domain $D_{r}$, which is bounded by $C$ and $C_{r}$, where $C$ is the boundary of $U$ and $L(r)$ the length of the image of $C_{r}$, then

$$
\begin{aligned}
& A(r)=\int_{r_{0}}^{r} \int_{C_{r}}\left(\frac{\left|x^{\prime}(\tau)\right|}{1+|x(\tau)|^{2}}\right)^{2} r d r d \theta+\text { const. } \\
& L(r)=\int_{C_{r}} \frac{\left|x^{\prime}(\tau)\right|}{1+|x(\tau)|^{2}} r d \theta
\end{aligned}
$$

where we write $r=r(t), \theta=\theta(t)$. Then by (2),

$$
(L(r))^{2} \leqq 2 \pi r \int_{\sigma_{r}}\left(\frac{x^{\prime}(\tau)}{1+|x(\tau)|^{2}}\right)^{2} r d \theta=2 \pi r \frac{d A(r)}{d r}
$$

Since $x(t)$ has an essential singularities on $E, x(t)$ takes in $U$ any value

2) G.C.Evans: Potentials and positively infinite singularities of harmonic functions. Monatshefte f. Math. u. Phys. 43(1936). 
infinitely often, except a set of values of logarithmic capacity zero $^{3}$, so that

$$
\lim _{r \rightarrow \infty} A(r)=\infty .
$$

Let $L(r)>(A(r))^{\frac{3}{4}}$ in a set of intervals $I_{\nu}=\left[\boldsymbol{r}_{\nu}, \boldsymbol{r}_{\nu}^{\prime}\right](\nu=1,2, \cdots)$, then from (3)

$$
\sum \int_{\nu} \frac{d r}{l r} \leqq 2 \pi \int^{\infty} \frac{d A(r)}{(A(r))^{3}}<\infty,
$$

hence there exists $r_{1}<r_{2}<\cdots<r_{n} \rightarrow \infty$, such that $L\left(r_{n t}\right) \leqq\left(A\left(r_{n}\right)\right)^{\frac{3}{4}}$, so that

$$
\frac{L\left(r_{n}\right)}{A\left(r_{n}\right)} \leqq \frac{1}{\left(A\left(r_{n}\right)\right)^{\frac{1}{4}}} \rightarrow 0 \text { as } r_{n} \rightarrow \infty
$$

Hence $F^{*}$ is regularly exhaustible in Ahlfors' sense ${ }^{4}$.

Let $C_{r}$ consists of $n=n(r)$ Jordan curves $C_{r}=C_{r}^{(1)}+\cdots+C_{r}{ }^{(i)}$ and let $L_{r}^{(i)}$ be the length of the image $\Lambda_{r}^{(i)}$ of $C_{r}^{(i)}$ on the $x$-sphere, then $L(r)=L_{r}^{(1)}+\cdots+$ $L_{r}{ }^{(n)}$.

Since by the hypothesis, $F^{*}$ is non-ramified relatively to $F$ and $x(t)$ has essential singularitites on $E$, we see easily that $A_{i}^{(i)}$ is not homotop null on $F$, so that $L_{i}^{(i)} \geqq L_{0}>0$, where $L_{0}$ is a fixed constant, hence

$$
L(r) \geqq L_{\ell} n(r) \text {. }
$$

Let $F_{r}^{*}$ be the image of $D_{r}$ on the $x$-sphere, then $F_{r} *$ is a covering surface of $F$, so that by Ahlfors' theorem on covering surfaces ${ }^{5)}$,

$$
\stackrel{+}{\rho}(r) \geqq \rho_{v} S(r)-h\left(L(r)+\lambda_{0}\right), \stackrel{+}{\rho}=\operatorname{Max}(\rho, 0),
$$

where $S(r)=A(r) / n \pi, n$ being the number of sheets of $F, \rho(r)$ is the Euler's characteristic of $F_{r}^{*}, \rho_{0}=2(p-1)>0$ is that of $F$ and $\lambda_{0}$ is the length of the image of $C$ and $h$ is a constant.

Since $\stackrel{+}{\rho}(r)=n(r)-2 \leqq n(r)$, we have from (6),

$$
\begin{gathered}
L(r) \\
L_{0} \geqq \rho_{0} S(r)-h\left(\boldsymbol{L}(r)+\lambda_{0}\right), \quad \text { or } \\
S(r) \leqq \frac{1}{\rho_{0}}\left(\frac{1}{L_{0}}+h\right)\left(L(r)+\lambda_{0}\right),
\end{gathered}
$$

which contradicts (5). Hence our theorem is proved.

3

Let $G$ be a group of linear transformations : $S_{\nu}=\frac{a_{\nu} z+b_{\nu}}{c_{v} z+d_{\nu}}(\nu=0,1,2, \cdots)$

3) S. KAMETANI : The exceptional values of functions with the set of capacity zero of essential singularities. Proc. Imp. Acad. 17(1941). M. TsuJI : Theory of meromorphic functions in a neighbourhood of a closed set of capacity zero. Jap. Journ. Math. 19 (1944-1948).

4) K. NOSHIRO : Contribution to the theory of the singularities of analytic functions Jap. Journ. Math. 19 (1944-1948).

5) L. AHLFoRs : Zur Theorie der Überlagerungsflächen. Acta Math. 65 (1935) 
of Schottky type, which contains at lest two generators. We call such a group a general linear group of Schottky type. Let $D_{0}$ be the fnndamental domain of $G$, which is bounded by $p(2 \leqq p \leqq \infty)$ pairs of Jordan curves $\boldsymbol{C}_{i}, \boldsymbol{C}_{i}^{\prime}(i=1,2, \cdots, p)$, where $\boldsymbol{C}_{i}, \boldsymbol{C}_{i}$ are equivalent by $\boldsymbol{G}$. If we apply all transformations of $G$ to $D_{0}$, then its equivalents $D_{v}$ cluster to a nondense perfect set $E$, which we call the singular set of $G$. Then Myrberg ${ }^{3)}$ proved the following theorem.

RHEOREM 3. The singular set E of a general linear group of Schottky type is of positive logarithmic capacity.

Proof. Let $D_{0}^{\prime}$ be the domain bounded by $C_{1}, C_{1}^{\prime}, C_{2}, C_{2}^{\prime}$ and $T_{1}, T_{2}$ be the transformations of $G$, such that $C_{1}^{\prime}=T_{1}\left(C_{1}\right), C_{2}^{\prime}=T_{2}\left(C_{2}\right)$ and let $G^{\prime}$ be the group generated by $\left\{T_{1}, T_{2}\right\}$. If we apply all transformations of $G^{\prime}$ to $D_{0}^{\prime}$, then its equivalents $D_{v}^{\prime}$ cluster, to a non-dense perfect set $E^{\prime} \subset E$. Now we consider $D_{0}^{\prime}$ as a closed Riemann surface $F$ of genus $p=2$, where equivalent point on $C_{i}, C_{i}^{\prime},(i=1,2)$ are considered as the same point of $F$. Then we have a non-ramified covering surface $F^{*}$ of $F$, where an equivalent point $z_{\nu}$ of $z_{0} \in D_{0}^{\prime}$ corresponds to the point $z_{0}$ of $F$. Hence by Theprem $2, E^{\prime}$ and hence $E$ is of positive logarithmic capacity.

Similarly we can prove the following theorem ${ }^{i)}$.

Theorem 4. Let $C_{1} \cdots, C_{p}(3 \leqq p \leqq \infty)$ be pircles on the $z$-plane, which lie outside each other. We invert $C_{i}$ inot $C_{i}$ and we perform inversions indefinitely, then we obtain infinitely many circles clustering to a non-dense perfect set $E$. Then $E$ is of positive logarithmic capacity.

Proof. We take three circles $C_{1}, C_{3}, C_{3}$ and $D_{0}$ be the domain bounded by these circles. We perform indefinitely inversions starting from $C_{1}, C_{2}$, $C_{3}$, then we have infinitely many circles clustering to a non-dense perfect set $E^{\prime} \subset E$. We take two same samples $D_{0}, D_{0}$ as $D_{0}$ and conncet them along $C_{i}(i=1,2,3)$, then we have a closed surface $F$ of genus $p=2$. Any point outside $E^{\prime}$ is equivalent to a point of $F$ by inversion, so that we have a nonramified covering surface $F^{*}$ of $F$, hence by Theorem $2, E^{\prime}$ and hence $E$ is of positive logarithmic capacity.

REMARK BY A. MORI.

The idea of the proof of Theorem 2 can be formulated in the following form, which is an analogue of Ahlfors's theorem for simply connected covering surfaces ${ }^{s}$.

Any non-ramified and unbounded (unberandet) open covering surface $F^{*}$ of planar character (or, more generally, of finite genus) of a closed basic surface $F$ of genus $\geqq 2$ is not regularly exhaustible in Ahlfors's sense.

Proof. Let $F_{1} \subset F_{2} \subset \cdots$ be an exhaustion of $F^{*}$. We assume that

6) P. J. Myrberg : Die Kapazität der singulären Menge der linearen Gruppen. Annales Acad. Fenn. Series A. Math- Phys. 10 (1941).

7) Myrberg. 1. c. 6).

8) Ahlfors. 1. c 5). 
ON THE UNIFORMIZATION OF ALGEBRAIC FUNCTION ETC.

281

the boundary of $F_{v}$ consists of $n_{v}$ rectifiable closed Jordan curves $\Lambda_{v}^{(i)}$ $\left(i=1,2, \cdots, n_{v}\right)$ on $F^{*}$. Let $A_{0}, A_{\nu}$ be the area of $F$ and $F_{\nu}$, and $L_{\nu}^{(i)}$ be the length of $\Lambda_{v}^{(i)}$ (both measured in a metric defined on $F$ ). We put $S_{\nu}=A_{\nu} / A_{0}, L_{\nu}=\sum_{i=1}^{n v} L_{v}^{(i)}$. Since $F^{*}$ is open and unbounded, we see easily that $S_{\nu} \rightarrow \infty$, and we have to prove that $L_{v} / S_{\nu}$ is bounded from zero.

If one of $\Lambda_{\nu}^{(i)}$ is null-homotop on $F^{*}$, it bounds a compact simply connetted domain $\Delta\left(\Lambda_{v}^{(i)}\right)$ on $F^{*}$. We add to $F_{\nu}$ all such domains and put $\bar{F}_{v}=F_{\nu}+\sum \Delta\left(\Lambda_{\nu}^{(i)}\right)$. Then, $\bar{F}_{1} \subset F_{2} \subset \cdots$ is also an exhaustion of $F^{*}$, and if $\bar{\Lambda}_{\nu}^{(i)}\left(j=1,2, \cdots, n_{v}\right), L_{v}^{(i)}, L_{v}$ and $S_{v}$ denote the corresponding curves and quantities, we have $S_{v} \leqq S_{v}, L_{v} \geqq L_{v}$. Further, let $\rho_{v}$ denote the Euler's characteristic of $F_{\nu}$.

Suppose that one of $\bar{\Lambda}_{\nu}^{(j)}$ is null-homotop on $F^{*}$. Then we see easily that $\bar{F}_{\nu}$ coincides with $\Delta\left(\Lambda_{v}^{\left(j^{\prime}\right)}\right)$ so that $\delta_{v}{ }^{+}=0$.

If none of $\Lambda_{i}^{\left(j^{\prime}\right)}$ is null-homotop on $F^{*}$, their projections on $F$ are not null-homotop on $F$, so that $L_{v}^{(j)} \geqq$ constr. $=L_{0}>0$. Then, we have $L_{\nu} \geqq n_{\nu} L_{0}$ and, since $F^{*}$ is of finite genus,

$$
\rho_{v^{+}}{ }^{+} \leqq \overline{n_{\nu}}+\text { constr. } \leqq \frac{\bar{L}_{v^{\prime}}}{\bar{L}_{3}}+\text { const. }
$$

Hence, in any case, Ahlfors' fundamental theorem gives $\left(\rho_{0}>0\right.$ benig the characteristic of $F$ )

$$
\frac{L_{v^{\prime}}}{L_{0}^{-}}+\text {cost. } \geqq \rho_{v}^{+} \geqq \rho_{0} S_{v^{\prime}}-h L_{v}
$$

so that

$$
\frac{L_{i^{\prime}}}{S_{\mathrm{r}^{\prime}}} \geqq \overline{\bar{L}} S_{S} \geqq \frac{\rho_{0} L_{0}}{1+h L_{0}}-O\left(\frac{1}{\bar{S}_{\nu}}\right) .
$$

Since $S_{\nu} \geqq S_{\nu} \rightarrow \infty$, we have $\lim \frac{L_{v}}{S_{v}}>0$, q. e. d.

Mathematical Institute, Tokyo University. 